\title{
Comparative effects of sesame oil and clove oil on growth performance, blood profile and immunity of rabbit (Oryctolagus cuniculus)
}

Memoona Iqbal $^{1 *}$, Razia Iqbal ${ }^{1}$, Mubashar Hussain ${ }^{1}$, Muhammad Faheem Malik $^{1}$, Abdul Razaq ${ }^{1}$ and Majid Hussain ${ }^{2}$

1. Department of Zoology, Faculty of Science, University of Gujrat, Punjab-Pakistan

2. Institute of Molecular Biology and Biotechnology, University of Lahore, Lahore-Pakistan

*Corresponding author's email: memoonaigbal1996@gmail.com

Citation

Memoona Iqbal, Razia Iqbal, Mubashar Hussain, Muhammad Faheem Malik, Abdul Razaq and Majid Hussain. Comparative effects of sesame oil and clove oil on growth performance, blood profile and immunity of rabbit (Oryctolagus cuniculus). Pure and Applied Biology. Vol. 11, Issue 2, pp491-504.

http://dx.doi.org/10.19045/bspab.2022.110048

Received: 27/05/2021 Revised: 01/07/2021

Accepted: 28/07/2021

Online First: 26/08/2021

\section{Abstract}

Essential oils are being used to improve health due to their high nutritive value. In this study, the comparative effects of Sesame oil and Clove oil were assessed on growth performance, blood profile and immunity of male rabbits. A total of 108 male rabbits were divided into 3 treatment groups; Treatment group A (Sesame oil), Treatment group B (Clove oil), Treatment group C (Sesame oil + Clove oil). Each treatment group was further divided into 4 subgroups according to dosage design of oils. The control group was untreated, while treatment dose for groups A and B was divided into 3 different concentrations; 0.1, 0.2 and 0.3ml/kilogram Body Weight. Whereas, the treatment concentrations for group $C$ were as; $0.1+0.1,0.2+0.2$ and $0.3+0.3 \mathrm{ml} / \mathrm{kilogram}$ Body Weight of oils for $1-1.5 \mathrm{~kg}$ of rabbits. The rabbits were managed in proper caging under controlled conditions of photoperiod (12 light: 12 dark cycles) $23 \pm 2^{\circ} \mathrm{C}$ of temperature. The selected dose concentration of each oil was administered orally and once a day for six weeks. The results showed that both oils had significantly enhanced the growth performance and reduced the Feed Conversion Ratio. The hematological parameters were increased in all treatment groups, respectively. The Enzyme-linked Immunosorbent Assay for Rabbit Hemorrhagic Disease Virus also showed a significant increase in antibody titer level. The study showed that Sesame oil and Clove oil as dietary supplements have significantly $(\mathrm{p}<0.05)$ improved overall performance of male rabbits. The current study clearly indicated that both oils could be a better choices for health purposes.

Keywords: Essential oils; ELISA; Hematology; Immune system

\section{Introduction}

Essential oils are aromatic liquids, with their biological activities like antibacterial, antifungal, anti-oxidant, and anti- inflammatory properties [1]. The oils are basically known to be as antibiotic growth agents, used in animal diet to induce positive 
impacts on growth performance, internal microbiota and health [2]. The bioactivity of the oils depends on the complex mixture of volatile and non-volatile compounds produced by the metabolism activity of aromatic natured plants [3].

Sesamum indicum is the oldest plant, belongs to the family Pedaliacecae and is mainly cultivated in tropical areas [4]. It is a stable edible oil with a high degree of unsaturation property, normally available as high-quality oil and rich in total phenolic content, flavonoids [5], lignans, tocopherol homologues, and phytosterols [6]. Oil is considered as a rich source of food because of calcium and phosphorus [7]. The seed contains $50-60 \%$ part of the oil, $18-25 \%$ of protein content, $13.5 \%$ of carbohydrates, and $5 \%$ of ash [8]. The oil is used to cure diseases and is helpful in lowering the concentration of cholesterol, controlling blood pressure, and dermatological disease management [9]. It also performs antioxidative, antihypersensitive, anti-cancer and antiimmunoregulatory activities [10]. It can cause allergic reactions but it is rare about $0.1 \%$ in one piece population [11]. It is used as solvent, skin softeners, margarines, pharmaceuticals and lubricants [12].

The Clove (Syzygium aromaticum) an aromatic flower bud of the tree that belongs to the family Myrtaceae, has the eugenol compound with beta-caryophyllene and has a lesser amount of the other constituents like benzyl alcohol in its oil [13]. The amount of eugenol is $78 \%$ with $13 \%$ beta-caryophyllene in the oil [14]. The chemical analysis shows that this oil has 36 components but 9 are with the highest concentrations [15]. It has antibacterial activity [16], a source of the new antifungal metabolites due to its phenolic constituents, the carvacrol, and the eugenol [17]. Clove oil has antioxidant properties and photocytotoxicity due to the eugenol $[18,19]$. It also has antimutagenic potential, so it shows antitumor action [20]. Clove oil is a popular medication for dentistry [21], headache, sore throat, respiratory system and digestive system aliment [22]. At very low concentrations, clove oil acts as anti-oxidant and anti-inflammatory property, but at higher concentrations, it can cause tissue damage [23]. It can enhance the learning and memory recall processes [24]. Clove oil has phytochemical extracts [25], when this oil is given intravenously at low concentration, it mainly helps to reduce the fever in rabbits [26]. Although, both oils perform various functions, however, this study was designed to estimate the comparative effects of Sesame oil and Clove oil on the growth performance, blood profile and immunity of rabbits.

\section{Materials and Methods Experimental animal}

Eight-week-old male rabbits (Oryctolagus cuniculus) with the weight of 1.0 to $1.5 \mathrm{~kg}$ were purchased from Ghazi Road Lahore Institute of Veterinary Research Lahore, Punjab, Pakistan during the month of February. These rabbits were vaccinated and acclimatized before experimentation for 15 days with optimum light i.e. 12 hour light and dark cycle and $23 \pm 2^{\circ} \mathrm{C}$ temperature [27]. Rabbits were kept in cages and were offered ad libitum feed and water, after every eight hours over the whole experiment. The selected concentration of essential oils was mixed in the soaked bread, given orally and once a day. To prevent from the disease and stress, the wasted food and feces of the rabbits were removed daily. Furthermore, bedding was also changed on daily basis to feel them comfortable, safe and warm.

\section{Essential oils and dosage design}

Oils of Sesame seeds and Clove buds were purchased from the local market. Rabbits were distributed into 3 treatment groups; Treatment group A (Sesame oil), Treatment group B (Clove oil) and Treatment Group C (Sesame oil + Clove oil). Each treatment group was further divided into 4 subgroups according to the dosage design of oils. The 
control group was untreated, while treatment dose for groups A and B was divided into 3 different concentrations; $0.1,0.2$ and $0.3 \mathrm{ml} / \mathrm{kg} \mathrm{BW}$. Whereas, the treatment concentrations for group $\mathrm{C}$ were as; $0.1+0.1$, $0.2+0.2$ and $0.3+0.3 \mathrm{ml} / \mathrm{kg} \mathrm{BW}$. The selected dose of each oil was given once a day for six weeks. To reduce error, three replications per treatment were implemented [28, 29].

\section{Growth performance (FCR)}

After every $15^{\text {th }}$ day of the experiment, the digital balance was used to check the weight of the rabbit in kilograms. The weight of each rabbit was checked after 12 hours of fasting, to avoid the addition of gut content in total body weight. The feed intake was fixed and measured for each group on daily basis. The feed consumption rate was also checked. The formula for feed conversion ratio was applied to get results [30].

FCR $=$ Total Feed Intake $/$ Total Weight Gain Blood sampling

At $15^{\text {th }}, 30^{\text {th }}$ and $45^{\text {th }}$ day of the research work, blood samples were collected through the jugular vein. About $2 \mathrm{ml}$ of blood sample was added into $3 \mathrm{ml} \mathrm{K}_{3}$ EDTA tubes for calculation of blood components. The same amount of blood was also added into the gel and clot activator tubes to extract the serum. After collecting the blood samples the specimens were treated with silver nitrate solution to halt the blood loss [31, 32].

\section{Hematological tests and ELISA}

The blood analysis was conducted in the Zoology Laboratory to check the levels of CBC count, via a Semi-Automatic Chemical Analyzer, model HKTE0112 Guangzhoun Hekang. Results were displayed numerically as well as graphically on the screen of the analyzer. Later on, these parameters were checked to identify the changes as compared to the control group $[31,33]$.

The serum was extracted by using centrifugation machine. Samples were centrifuged at the speed of $1600 \mathrm{xg}$ for 15 minutes at $4^{\circ} \mathrm{C}$ to obtain the serum from the blood and separated in microtubes for test analysis [34]. To check the humoral responses, an indirect ELISA-Array assay was performed in the Zoology Laboratory. Then serum samples were used to run the ELISA test. The antibodies present in the serum sample were attached to colorless substrate and color product was obtained at the end of the test. So, the antigen and antibody combination was helpful to access the results during the ELISA procedure [35].

\section{Data analysis}

All the mean standard values were obtained with the help of the SPSS (Statistical Packages for Social Science). Standard Error Mean $( \pm$ S.E.M) for each of parameters. Moreover, Variation between groups was detected by the ANOVA test along with post hoc test (Tukey HSD). The extent of variation found in the groups. The $\mathrm{P}<0.05$ value was deliberated as statistically significant.

\section{Results}

\section{Body weight and FCR}

When Sesame oil was orally administered to rabbits, it was observed that body weight was significantly $(\mathrm{p}<0.05)$ increased (Fig. 1) at concentration $0.3 \mathrm{ml} / \mathrm{kg} \mathrm{BW}$ with mean value of $4.84 \pm 0.02 \mathrm{~kg}$ (Table 1) and the FCR was significantly decreased (Fig. 2) with this mean value $4.05 \pm 0.04$.

When Clove oil was orally administered to rabbits, it was examined that body weight increased (Fig. 3) at $0.2 \mathrm{ml} / \mathrm{kg} \quad \mathrm{BW}$ concentration with mean value of $4.35 \pm 0.00$ $\mathrm{kg}$ (Table 2) and the FCR was significantly decreased (Fig. 4) with the mean value $4.05 \pm 0.04$.

When the combination of both oils $\mathrm{SO}+\mathrm{CO}$ was given to rabbits, it was observed that it has exhibited maximum weight gain (Fig. 5) at concentrations $0.3+0.3 \mathrm{ml} / \mathrm{kg} \mathrm{BW}$ with a mean value of $5.45 \pm 0.01 \mathrm{~kg}$ (Table 3 ) and with decrease in FCR (Fig. 6) with a mean value of $3.80 \pm 0.05$ for all treated groups as compared to untreated groups. 


\section{Blood profile}

The level of blood profile components was significantly increased (Table 4) throughout the study. Sesame oil has increased the blood components at concentration $0.3 \mathrm{ml} / \mathrm{kg} \mathrm{BW}$. The mean values of $\mathrm{Hb}$ level $(14.25 \pm 0.38$ $\mathrm{g} / \mathrm{dl})$, RBC's count $\left(8.06 \pm 0.1210^{\wedge} 12 / 1\right)$, HCT level (53.66 $\pm 0.88 \%), \mathrm{MCV}(83.66 \pm$ $0.88 \mathrm{fL})$, MCH level $(41.00 \pm 1.52 \mathrm{pg})$, MCHC level $(46.33 \pm 0.88 \mathrm{~g} / \mathrm{dl})$, WBC's count $\left(11.86 \pm 0.1310^{\wedge} 9 / 1\right)$ and platelets number (342.33 \pm 0.88 10^9/1) have showed the increase than the control group.

Clove oil has improved the blood profile at concentration $0.2 \mathrm{ml} / \mathrm{kg} \mathrm{BW}$. The average values for $\mathrm{Hb}$ level $(14.06 \pm 0.46 \mathrm{~g} / \mathrm{dl})$, RBC's count $\left(7.83 \pm 0.0310^{\wedge} 12 / 1\right)$, HCT level $(53.00 \pm 2.30 \%)$, MCV $(83.66 \pm 0.88$ $\mathrm{fL}), \mathrm{MCH}$ level (36.66 $\pm 0.33 \mathrm{pg}$ ), MCHC level $(41.33 \pm 1.20 \mathrm{~g} / \mathrm{dl})$, WBC's count $\left(13.43 \pm 0.2310^{\wedge} 9 / 1\right)$ and platelets number $\left(392.00 \pm 3.5110^{\wedge} 9 / \mathrm{l}\right)$ have exhibit the increase as compared to the control group.
The hematological parameters of $\mathrm{SO}+\mathrm{CO}$ treated groups were higher at concentrations $0.3+0.3 \mathrm{ml} / \mathrm{kg} \mathrm{BW}$ than other treatment and control group with mean values such as $\mathrm{Hb}$ level $(16.03 \pm 0.14 \mathrm{~g} / \mathrm{dl}), \mathrm{RBC}$ 's count $(9.76$ $\left.\pm 0.1410^{\wedge} 12 / 1\right)$, HCT level (58.66 $\left.\pm 1.76 \%\right)$, MCV (95.33 $\pm 1.76 \mathrm{fL}), \mathrm{MCH}$ level (47.66 \pm $0.88 \mathrm{pg}), \mathrm{MCHC}$ level $(58.66 \pm 2.60 \mathrm{~g} / \mathrm{dl})$, WBC's count $\left(14.83 \pm 0.6010^{\wedge} 9 / 1\right)$ and platelets number $(562.66 \pm 3.17$ 10^9/1).

\section{Immunity}

Sesame oil has shown maximum increase (Fig. 7) in immunity of rabbits (Table 5) at $0.3 \mathrm{ml} / \mathrm{kg}$ BW concentration with mean antibody titer value of $6024.66 \pm 85.10$ during whole experiment. Clove oil has displayed an increase in immunity (Fig. 8) at $0.2 \mathrm{ml} / \mathrm{kg}$ BW concentration (Table 6) with mean antibody titer value of $5166.00 \pm$ 140.26. The SO + CO has enhanced the immunity (Fig. 9) at $0.3+0.3 \mathrm{ml} / \mathrm{kg} \mathrm{BW}$ concentrations (Table 7) with the mean antibody titer value of $6425.00 \pm 87.36$ to premier level than other groups.

Table 1. Effect of Sesame Oil on Body Weight of rabbits at $15^{\text {th }}, 30^{\text {th }}$ and $45^{\text {th }}$ Days (Mean \pm S.E.M)

\begin{tabular}{|c|c|c|c|c|}
\hline \multirow{2}{*}{$\begin{array}{c}\text { Duration } \\
\text { (in days) }\end{array}$} & \multicolumn{4}{|c|}{ Treatment } \\
\cline { 2 - 5 } & $\mathbf{( C o n t r o l )}^{\mathrm{C}}$ & $\mathbf{( \mathbf { 0 . 1 m l } / \mathbf { k g ~ B W } )}$ & $\mathbf{( \mathbf { 0 . 2 } \mathbf { m l } / \mathbf { k g ~ B W } )}$ & $\mathbf{( \mathbf { 0 . 3 m l } / \mathbf { k g ~ B W } )}$ \\
\hline 15 & $2.15 \pm 0.02^{\mathrm{a}}$ & $2.32 \pm 0.04^{\mathrm{b}}$ & $2.54 \pm 0.02^{\mathrm{c}}$ & $2.62 \pm 0.01^{\mathrm{d}}$ \\
\hline 30 & $2.90 \pm 0.02^{\mathrm{a}}$ & $3.35 \pm 0.03^{\mathrm{b}}$ & $3.58 \pm 0.04^{\mathrm{c}}$ & $3.78 \pm 0.02^{\mathrm{d}}$ \\
\hline 45 & $3.54 \pm 0.02^{\mathrm{a}}$ & $4.35 \pm 0.03^{\mathrm{b}}$ & $4.64 \pm 0.04^{\mathrm{c}}$ & $4.84 \pm 0.02^{\mathrm{d}}$ \\
\hline
\end{tabular}

Means with different superscript in a row are significantly different from one another $(\mathrm{p} \leq 0.05)$ Tukey's Test

Table 2. Effect of Clove Oil on Body Weight of rabbits at $15^{\text {th }}, 3^{\text {th }}$ and $45^{\text {th }}$ Days (Mean \pm S.E.M)

\begin{tabular}{|c|c|c|c|c|}
\hline \multirow{2}{*}{$\begin{array}{c}\text { Duration } \\
\text { (in days) }\end{array}$} & \multicolumn{4}{|c|}{ Treatment } \\
\cline { 2 - 5 } & $\mathbf{T}_{\mathbf{o}}(\mathbf{C o n t r o l})$ & $\mathbf{( 0 . 1 \mathbf { m l } / \mathbf { k g ~ B W } )}$ & $\mathbf{( \mathbf { 0 . 2 m l } / \mathbf { k g ~ B W } )}$ & $\mathbf{( \mathbf { 0 . 3 m l } / \mathbf { k g ~ B W } )}$ \\
\hline 15 & $2.15 \pm 0.02^{\mathrm{a}}$ & $2.30 \pm 0.02^{\mathrm{c}}$ & $2.54 \pm 0.04^{\mathrm{d}}$ & $2.43 \pm 0.03^{\mathrm{b}}$ \\
\hline 30 & $2.90 \pm 0.02^{\mathrm{a}}$ & $3.51 \pm 0.02^{\mathrm{c}}$ & $3.71 \pm 0.01^{\mathrm{d}}$ & $3.43 \pm 0.01^{\mathrm{b}}$ \\
\hline 45 & $3.54 \pm 0.02^{\mathrm{a}}$ & $4.20 \pm 0.02^{\mathrm{c}}$ & $4.35 \pm 0.00^{\mathrm{d}}$ & $3.90 \pm 0.04^{\mathrm{b}}$ \\
\hline
\end{tabular}

Means with different superscript in a row are significantly different from one another ( $\mathrm{p} \leq 0.05)$ Tukey's Test 
Table 3. Effects of Sesame Oil + Clove Oil on Body Weight of rabbits at $15^{\text {th }}, 30^{\text {th }}$ and $45^{\text {th }}$ Days (Mean \pm S.E.M)

\begin{tabular}{|c|c|c|c|c|}
\hline \multirow[b]{2}{*}{$\begin{array}{c}\text { Duration } \\
\text { (in days) }\end{array}$} & \multicolumn{4}{|c|}{ Treatment } \\
\hline & (Control) & $\begin{array}{c}(0.1+0.1 \mathrm{ml} / \mathrm{kg} \\
\text { BW) }\end{array}$ & $\begin{array}{c}(0.2+0.2 \mathrm{ml} / \mathrm{kg} \\
\text { BW) }\end{array}$ & $(0.3+0.3 \mathrm{ml} / \mathrm{kg} \mathrm{BW})$ \\
\hline 15 & $2.15 \pm 0.02^{\mathrm{a}}$ & $2.49 \pm 0.05^{\mathrm{b}}$ & $2.72 \pm 0.04^{\mathrm{c}}$ & $3.00 \pm 0.08^{\mathrm{d}}$ \\
\hline 30 & $2.90 \pm 0.02^{\mathrm{a}}$ & $3.93 \pm 0.04^{b}$ & $4.30 \pm 0.02^{\mathrm{c}}$ & $4.61 \pm 0.07^{\mathrm{d}}$ \\
\hline 45 & $3.54 \pm 0.02^{\mathrm{a}}$ & $4.69 \pm 0.02^{b}$ & $4.89 \pm 0.05^{\mathrm{c}}$ & $5.45 \pm 0.01^{\mathrm{d}}$ \\
\hline
\end{tabular}

Means with different superscript in a row are significantly different from one another $(\mathrm{p} \leq 0.05)$ Tukey’s Test

Table 4. Maximum Effect of Sesame Oil, Clove Oil, Sesame Oil + Clove Oil on Blood Parameters of Rabbits at following Concentrations with $15^{\text {th }}, 30^{\text {th }}$ and $45^{\text {th }}$ Days duration

\begin{tabular}{|c|c|c|c|c|c|}
\hline \multirow{3}{*}{ Parameters } & \multirow{3}{*}{ Duration } & \multicolumn{4}{|c|}{ Max Effect at following Conc. of Oils } \\
\hline & & & Sesame Oil & Clove Oil & $\mathrm{SO}+\mathrm{CO}$ \\
\hline & & Control & $0.3 \mathrm{ml} / \mathrm{kg} \mathrm{BW}$ & $0.2 \mathrm{ml} / \mathrm{kg} \mathrm{BW}$ & $.3+0.3 \mathrm{ml} / \mathrm{kg} \mathrm{BW}$ \\
\hline $\mathrm{Hb}(\mathrm{g} / \mathrm{dL})$ & \multirow{8}{*}{$15^{\text {th }}$ Day } & $10.50 \pm 0.28^{\mathrm{a}}$ & $13.58 \pm 0.30^{\mathrm{c}}$ & $12.06 \pm 0.08^{\mathrm{b}}$ & $13.33 \pm 0.42^{\mathrm{d}}$ \\
\hline RBCs $(x 103 / \mu 1)$ & & $5.70 \pm 0.05^{\mathrm{a}}$ & $6.73 \pm 0.12^{\mathrm{d}}$ & $6.13 \pm 0.18^{\mathrm{c}}$ & $7.13 \pm 0.38^{d}$ \\
\hline $\mathrm{HCT}(\%)$ & & $41.00 \pm 0.57^{\mathrm{a}}$ & $45.33 \pm 0.33^{\mathrm{c}}$ & $42.00 \pm 1.52^{\mathrm{c}}$ & $48.66 \pm 0.33^{\mathrm{d}}$ \\
\hline MCV (fL) & & $61.00 \pm 0.57^{\mathrm{a}}$ & $66.00 \pm 0.57^{\mathrm{c}}$ & $67.00 \pm 1.15^{\mathrm{b}}$ & $68.00 \pm 0.57^{\mathrm{c}}$ \\
\hline $\mathrm{MCH}(\mathrm{pg})$ & & $18.00 \pm 0.57^{\mathrm{a}}$ & $23.33 \pm 1.20^{\mathrm{d}}$ & $25.66 \pm 0.88^{d}$ & $31.33 \pm 1.76^{\mathrm{d}}$ \\
\hline $\mathrm{MCHC}(\mathrm{g} / \mathrm{dL})$ & & $30.00 \pm 0.57^{\mathrm{a}}$ & $34.66 \pm 0.66^{\mathrm{c}}$ & $37.33 \pm 1.20^{\mathrm{c}}$ & $40.66 \pm 1.45^{\mathrm{c}}$ \\
\hline $\begin{array}{l}\text { Platelets } \\
(\mathrm{x} 103 / \mu \mathrm{l})\end{array}$ & & $227.33 \pm 3.71^{\mathrm{a}}$ & $241.33 \pm 1.85^{\mathrm{c}}$ & $305.00 \pm 6.02^{c}$ & $376.33 \pm 8.56^{\mathrm{d}}$ \\
\hline WBCs $(x 103 / \mu 1)$ & & $8.00 \pm 0.57^{\mathrm{a}, \mathrm{b}}$ & $8.30 \pm 0.35^{\mathrm{b}}$ & $9.00 \pm 0.35^{\mathrm{c}}$ & $11.16 \pm 0.21^{\mathrm{c}}$ \\
\hline $\mathrm{Hb}(\mathrm{g} / \mathrm{dL})$ & \multirow{8}{*}{ 30 ${ }^{\text {th }}$ Day } & $11.50 \pm 0.28^{\mathrm{a}}$ & $13.56 \pm 0.31^{\mathrm{c}}$ & $12.93 \pm 0.26^{\mathrm{b}}$ & $15.06 \pm 0.17^{\mathrm{d}}$ \\
\hline RBCs $(x 103 / \mu 1)$ & & $5.90 \pm 0.05^{\mathrm{a}}$ & $6.83 \pm 0.08^{\mathrm{d}}$ & $7.06 \pm 0.34^{\mathrm{c}}$ & $8.10 \pm 0.20^{\mathrm{d}}$ \\
\hline $\mathrm{HCT}(\%)$ & & $44.00 \pm 0.57^{\mathrm{a}}$ & $51.00 \pm 0.57^{\mathrm{c}}$ & $51.33 \pm 1.76^{\mathrm{c}}$ & $52.33 \pm 0.88^{d}$ \\
\hline MCV (fL) & & $66.66 \pm 0.88^{a}$ & $76.00 \pm 0.57^{\mathrm{c}}$ & $77.33 \pm 2.90^{\mathrm{b}}$ & $86.33 \pm 1.45^{\mathrm{c}}$ \\
\hline $\mathrm{MCH}(\mathrm{pg})$ & & $21.66 \pm 0.33^{\mathrm{a}}$ & $29.66 \pm 0.88^{d}$ & $32.66 \pm 0.33^{d}$ & $37.33 \pm 0.88^{d}$ \\
\hline $\mathrm{MCHC}(\mathrm{g} / \mathrm{dL})$ & & $33.00 \pm 0.57^{\mathrm{a}}$ & $38.33 \pm 0.88^{c}$ & $40.00 \pm 1.52^{\mathrm{c}}$ & $47.66 \pm 0.88^{c}$ \\
\hline $\begin{array}{l}\text { Platelets } \\
(\mathrm{x} 103 / \mu \mathrm{l})\end{array}$ & & $292.66 \pm 2.60^{a}$ & $331.00 \pm 1.52^{\mathrm{c}}$ & $378.33 \pm 6.06^{c}$ & $443.66 \pm 16.18^{\mathrm{d}}$ \\
\hline $\begin{array}{l}\text { 9WBCs } \\
(\mathrm{x} 103 / \mu \mathrm{l})\end{array}$ & & $9.33 \pm 0.88^{a, b}$ & $10.36 \pm 0.18^{b}$ & $11.40 \pm 0.20^{\mathrm{c}}$ & $13.93 \pm 0.26^{\mathrm{c}}$ \\
\hline $\mathrm{Hb}(\mathrm{g} / \mathrm{dL})$ & \multirow{8}{*}{$4^{\text {th }}$ Day } & $12.00 \pm 0.28^{a}$ & $14.25 \pm 0.38^{c}$ & $14.06 \pm 0.46^{\mathrm{b}}$ & $16.03 \pm 0.14^{\mathrm{d}}$ \\
\hline $\operatorname{RBCs}(x 103 / \mu)$ & & $6.36 \pm 0.08^{\mathrm{a}}$ & $8.06 \pm 0.12^{\mathrm{d}}$ & $7.83 \pm 0.03^{\mathrm{c}}$ & $9.76 \pm 0.14^{\mathrm{d}}$ \\
\hline HCT (\%) & & $47.00 \pm 0.57^{\mathrm{a}}$ & $53.66 \pm 0.88^{c}$ & $53.00 \pm 2.30^{\mathrm{c}}$ & $58.66 \pm 1.76^{\mathrm{d}}$ \\
\hline MCV (fL) & & $76.66 \pm 1.45^{\mathrm{a}}$ & $83.66 \pm 0.88^{c}$ & $83.66 \pm 0.88^{b}$ & $95.33 \pm 1.76^{\mathrm{c}}$ \\
\hline $\mathrm{MCH}(\mathrm{pg})$ & & $26.33 \pm 0.88^{\mathrm{a}}$ & $41.00 \pm 1.52^{\mathrm{d}}$ & $36.66 \pm 0.33^{d}$ & $47.66 \pm 0.88^{d}$ \\
\hline $\mathrm{MCHC}(\mathrm{g} / \mathrm{dL})$ & & $35.00 \pm 0.57^{\mathrm{a}}$ & $46.33 \pm 0.88^{c}$ & $41.33 \pm 1.20^{\mathrm{c}}$ & $58.66 \pm 2.60^{\mathrm{c}}$ \\
\hline $\begin{array}{l}\text { Platelets } \\
(\mathrm{x} 103 / \mu \mathrm{l})\end{array}$ & & $323.00 \pm 4.35^{\mathrm{a}}$ & $342.33 \pm 0.88^{c}$ & $392.00 \pm 3.51^{\mathrm{c}}$ & $562.66 \pm 3.17^{d}$ \\
\hline WBCs $(x 103 / \mu 1)$ & & $11.00 \pm 0.57^{\mathrm{a}, \mathrm{b}}$ & $11.86 \pm 0.13^{\mathrm{b}}$ & $13.43 \pm 0.23^{\mathrm{c}}$ & $14.83 \pm 0.60^{\mathrm{c}}$ \\
\hline
\end{tabular}

Means with different superscript in a row are significantly different from one another $(\mathrm{p} \leq 0.05)$ Tukey’s Test 
Table 5. Effect of Sesame Oil on Antibody Titer Level against Rabbit Hemorrhagic Disease Virus (RHDV) of Rabbits at $15^{\text {th }}, 30^{\text {th }}$ and $45^{\text {th }}$ Days (Mean \pm S.E.M)

\begin{tabular}{|c|c|c|c|c|}
\hline \multirow{2}{*}{$\begin{array}{c}\text { Duration } \\
\text { (in days) }\end{array}$} & \multicolumn{4}{|c|}{ Treatment } \\
\cline { 2 - 5 } & $\mathbf{( C o n t r o l )}^{\mathrm{C}}$ & $\mathbf{( \mathbf { 0 . 1 } \mathbf { m } / \mathbf { k g ~ B W } )}$ & $\mathbf{( \mathbf { 0 . 2 } \mathbf { m l } / \mathbf { k g ~ B W } )}$ & $\mathbf{( \mathbf { 0 . 3 m l } / \mathbf { k g ~ B W } )}$ \\
\hline 15 & $3220.66 \pm 82.84^{\mathrm{a}}$ & $4091.33 \pm 112.29^{\mathrm{a}}$ & $4458.00 \pm 142.12^{\mathrm{b}}$ & $4858.00 \pm 142.12^{\mathrm{c}}$ \\
\hline 30 & $3760.66 \pm 32.05^{\mathrm{a}}$ & $4350.00 \pm 79.99^{\mathrm{a}}$ & $4658.00 \pm 199.58^{\mathrm{b}}$ & $5124.66 \pm 142.16^{\mathrm{c}}$ \\
\hline 45 & $4105.66 \pm 34.91^{\mathrm{a}}$ & $4588.33 \pm 54.65^{\mathrm{a}}$ & $5258.00 \pm 142.12^{\mathrm{b}}$ & $6024.66 \pm 85.10^{\mathrm{c}}$ \\
\hline
\end{tabular}

Means with different superscript in a row are significantly different from one another $(\mathrm{p} \leq 0.05)$ Tukey's Test

Table 6. Effect of Clove Oil on Antibody Titer Level against Rabbit Hemorrhagic Disease Virus (RHDV) of Rabbits at $15^{\text {th }}, 3^{\text {th }}$ and $45^{\text {th }}$ Days (Mean \pm S.E.M)

\begin{tabular}{|c|c|c|c|c|}
\hline \multirow{2}{*}{$\begin{array}{c}\text { Duration } \\
\text { (in days) }\end{array}$} & \multicolumn{4}{|c|}{ Treatment } \\
\cline { 2 - 5 } & $\mathbf{( C o n t r o l )}^{\mathrm{C}}$ & $\mathbf{( 0 . 1 m l} / \mathbf{k g ~ B W})$ & $\mathbf{( \mathbf { 0 . 2 } m / / \mathbf { k g ~ B W } )}$ & $\mathbf{( \mathbf { 0 . 3 m l } / \mathbf { k g ~ B W } )}$ \\
\hline 15 & $3220.66 \pm 82.84^{\mathrm{a}}$ & $4026.33 \pm 34.93^{\mathrm{b}}$ & $4222.33 \pm 37.33^{\mathrm{c}}$ & $4556.66 \pm 88.63^{\mathrm{c}}$ \\
\hline 30 & $3760.66 \pm 32.05^{\mathrm{a}}$ & $4328.33 \pm 88.59^{\mathrm{b}}$ & $4791.66 \pm 60.06^{\mathrm{c}}$ & $4563.00 \pm 34.00^{\mathrm{c}}$ \\
\hline 45 & $4105.66 \pm 34.91^{\mathrm{a}}$ & $4493.00 \pm 54.58^{\mathrm{b}}$ & $5166.00 \pm 140.26^{\mathrm{c}}$ & $4993.33 \pm 253.66^{\mathrm{c}}$ \\
\hline
\end{tabular}

Means with different superscript in a row are significantly different from one another $(\mathrm{p} \leq 0.05)$ Tukey's Test

Table 7. Effects of Sesame Oil + Clove Oil on Antibody Titer Level against Rabbit Hemorrhagic Disease Virus (RHDV) of Rabbits at $15^{\text {th }}, 30^{\text {th }}$ and $45^{\text {th }}$ Days (Mean \pm S.E.M)

\begin{tabular}{|c|c|c|c|c|}
\hline \multirow[b]{2}{*}{$\begin{array}{l}\text { Duration } \\
\text { (in days) }\end{array}$} & \multicolumn{4}{|c|}{ Treatment } \\
\hline & (Control) & $\begin{array}{c}(0.1+0.1 \mathrm{ml} / \mathrm{kg} \\
\text { BW) }\end{array}$ & $\begin{array}{c}(0.2+0.2 \mathrm{ml} / \mathrm{kg} \\
\text { BW) }\end{array}$ & $\begin{array}{c}(0.3+0.3 \mathrm{ml} / \mathrm{kg} \\
\text { BW) }\end{array}$ \\
\hline 15 & $3220.66 \pm 82.84^{\mathrm{a}}$ & $4360.00 \pm 87.57^{b}$ & $4756.33 \pm 88.95^{\mathrm{c}}$ & $5123.33 \pm 87.31^{d}$ \\
\hline 30 & $3760.66 \pm 32.05^{\mathrm{a}}$ & $4597.33 \pm 115.75^{b}$ & $5225.00 \pm 112.60^{c}$ & $5759.66 \pm 91.78^{d}$ \\
\hline 45 & $4105.66 \pm 34.91^{\mathrm{a}}$ & $4930.33 \pm 145.64^{b}$ & $5592.00 \pm 117.24^{\mathrm{c}}$ & $6425.00 \pm 87.36^{\mathrm{d}}$ \\
\hline
\end{tabular}

Means with different superscript in a row are significantly different from one another ( $\mathrm{p} \leq 0.05)$ Tukey’s Test

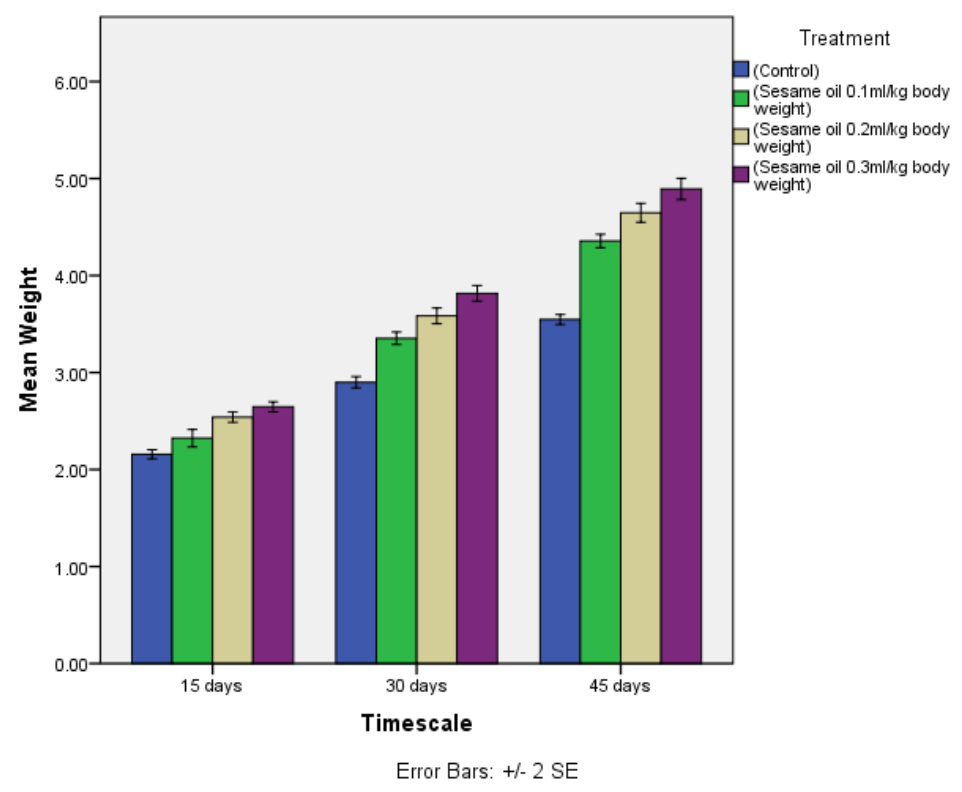

Figure 1. Concentration and Time Dependent Effect of Sesame Oil on Body Weight of Rabbits at Different Time-Scales $\left(15^{\text {th }}, 30^{\text {th }}\right.$ and $45^{\text {th }}$ Days $)$ 


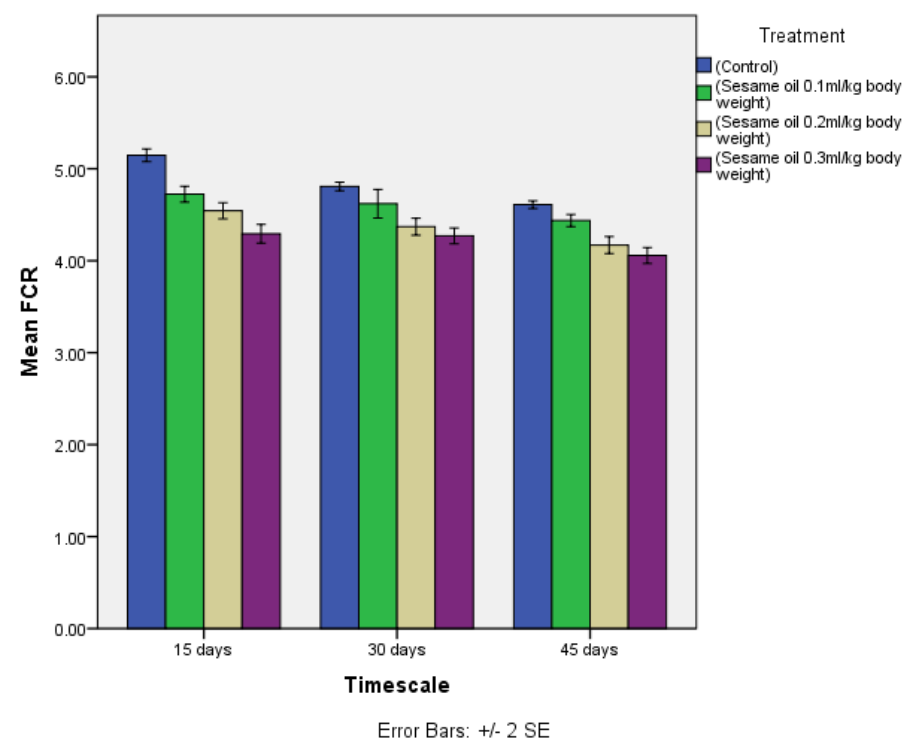

Figure 2. Concentration and Time Dependent Effect of Sesame Oil on Feed Conversion Ratio (FCR) of Rabbits at Different Time-Scales $\left(15^{\text {th }}, 30^{\text {th }}\right.$ and $45^{\text {th }}$ Days)

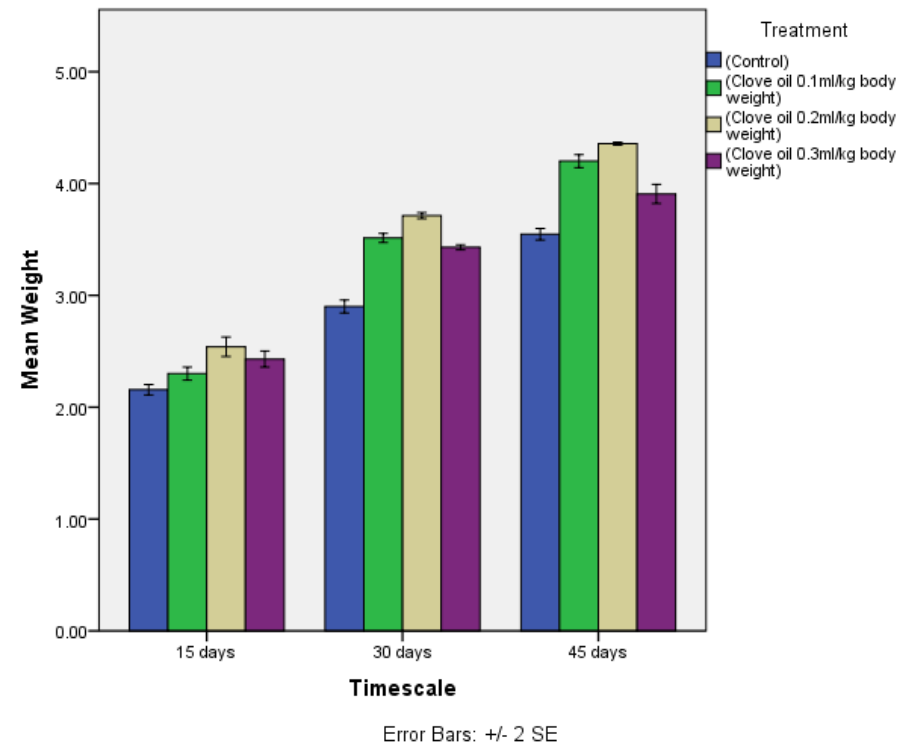

Figure 3. Concentration and Time Dependent Effect of Clove Oil on Body Weight of Rabbits at Different Time-Scales $\left(15^{\text {th }}, 30^{\text {th }}\right.$ and $45^{\text {th }}$ Days $)$ 
lqbal et al.

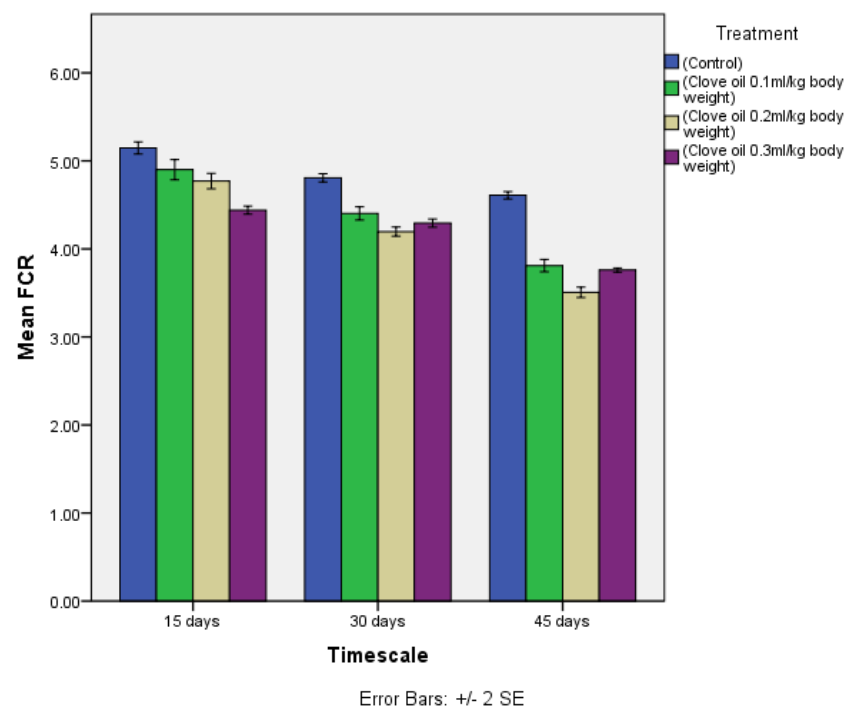

Figure 4. Concentration and Time Dependent Effect of Clove Oil on Feed Conversion Ratio (FCR) of Rabbits at Different Time-Scales $\left(15^{\text {th }}, 30^{\text {th }}\right.$ and $45^{\text {th }}$ Days)

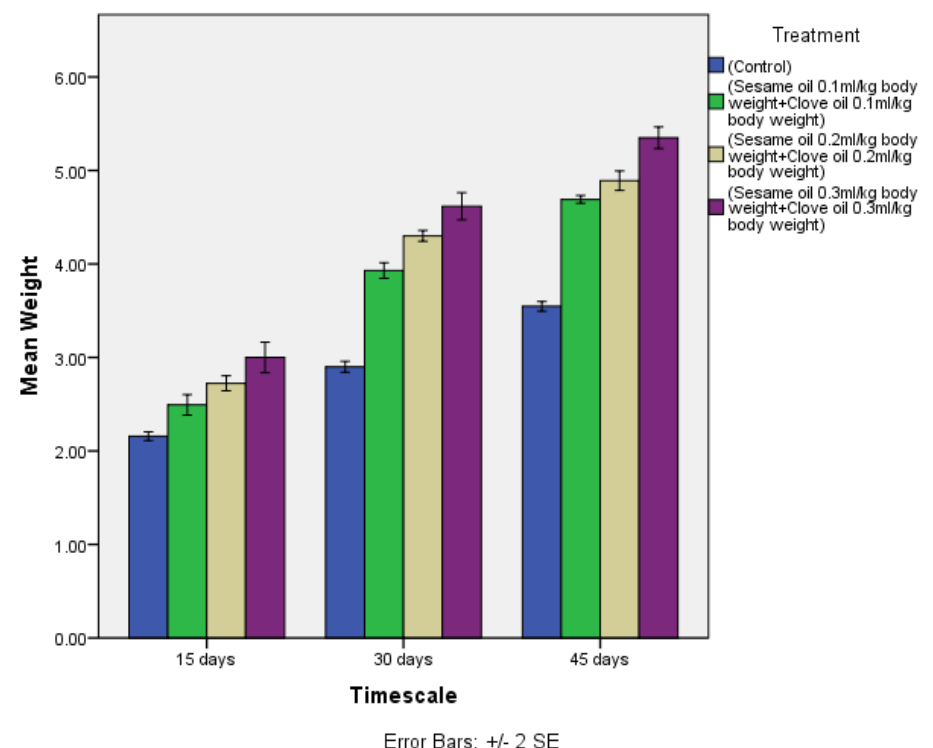

Figure 5. Concentration and Time Dependent Effects of Sesame Oil + Clove Oil on Body Weight of Rabbits at Different Time-Scales $\left(15^{\text {th }}, 30^{\text {th }}\right.$ and $45^{\text {th }}$ Days) 


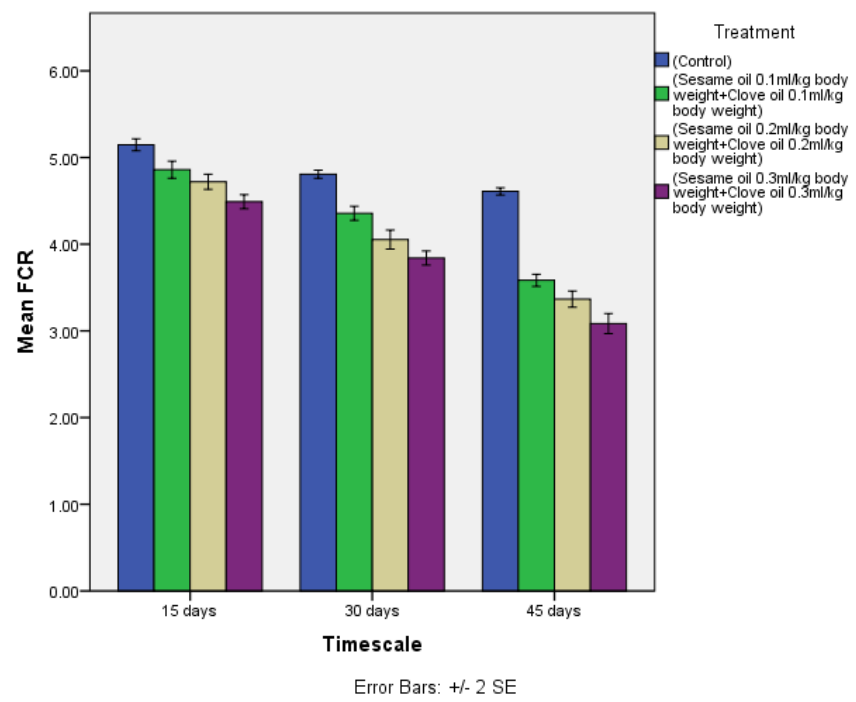

Figure 6. Concentration and Time Dependent Effects of Sesame Oil + Clove Oil on Feed Conversion Ratio (FCR) of Rabbits at Different Time-Scales $\left(15^{\text {th }}, 30^{\text {th }}\right.$ and $45^{\text {th }}$ Days)

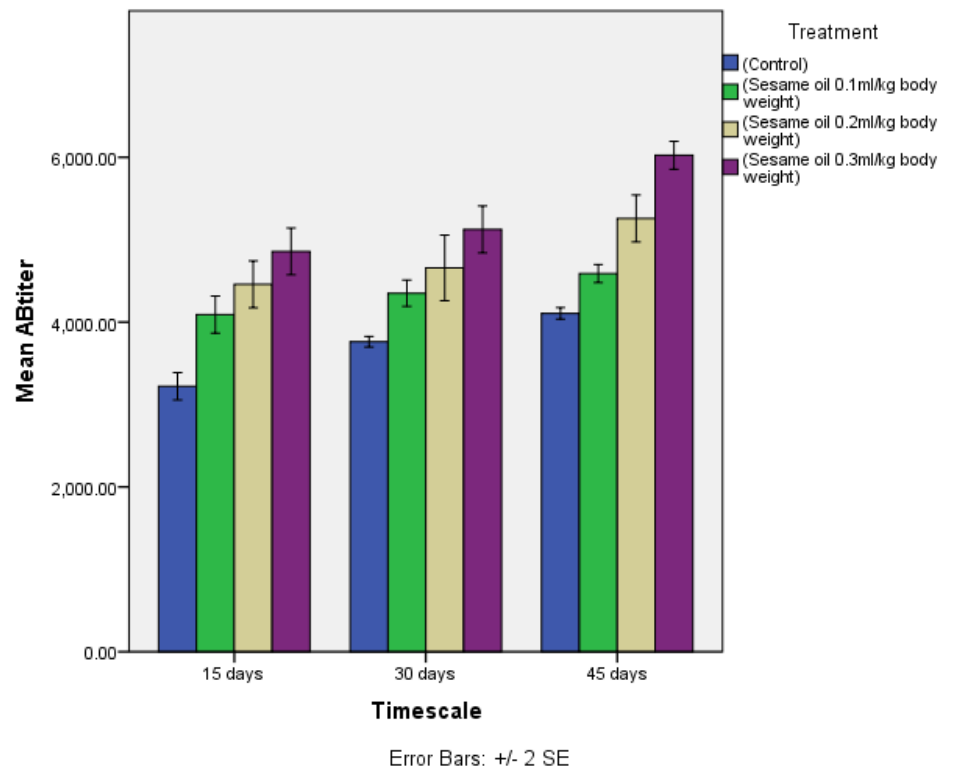

Figure 7. Concentration and Time Dependent Effect of Sesame Oil on Antibody Titer level against Rabbit Hemorrhagic Disease Virus (RHDV) of Rabbits at Different Time-Scales $\left(15^{\text {th }}, 3^{\text {th }}\right.$ and $45^{\text {th }}$ Days) 
lqbal et al.

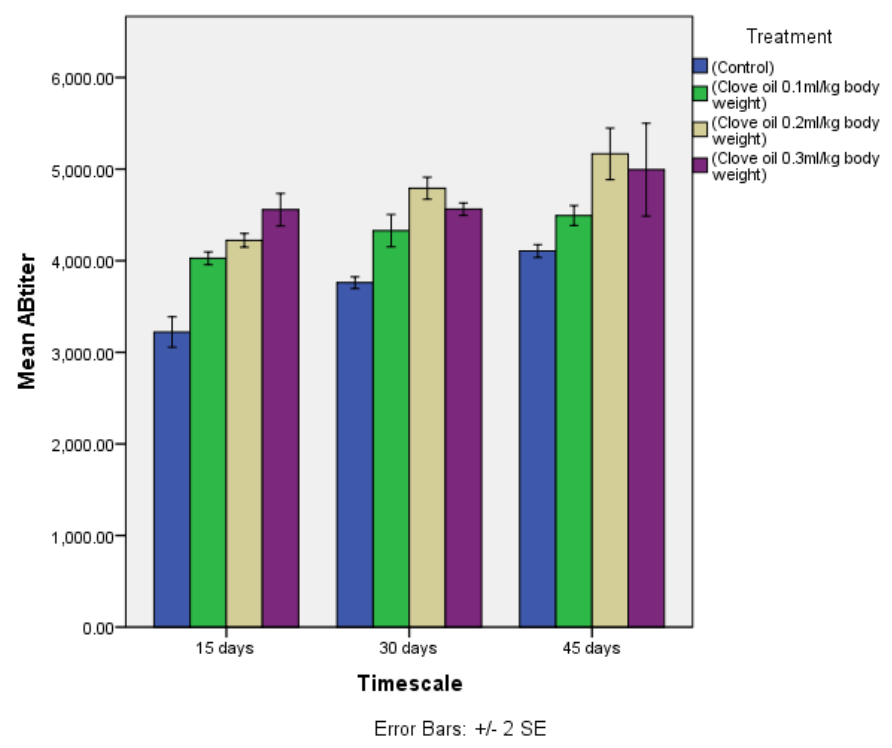

Figure 8. Concentration and Time Dependent Effect of Clove Oil on Antibody Titer level against Rabbit Hemorrhagic Disease Virus (RHDV) of Rabbits at Different Time-Scales $\left(15^{\text {th }}, 3^{\text {th }}\right.$ and $45^{\text {th }}$ Days $)$

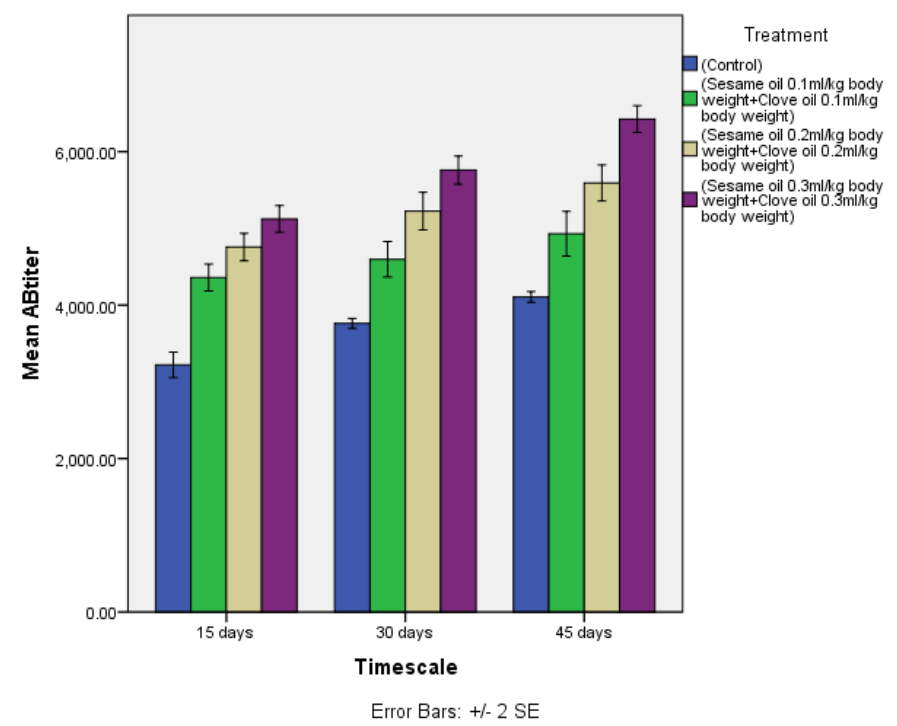

Figure 9. Concentration and Time Dependent Effects of Sesame Oil + Clove Oil on Antibody Titer level against Rabbit Hemorrhagic Disease Virus (RHDV) of Rabbits at Different TimeScales $\left(15^{\text {th }}, 30^{\text {th }}\right.$ and $45^{\text {th }}$ Days $)$

\section{Discussion}

A number of studies correlate with my findings to evaluate the effect of Sesame oil. It has been observed that Sesame oil proceeded as a good substitute of protein source with $30 \%$ amount of feed for growing rabbits and significantly increased body weight [36]. It was also indicated that Sesame oil has increased the blood constituents as red blood cells and white blood cells at dose concentrations $0.5 \mathrm{ml}, 1 \mathrm{ml}$ and $2 \mathrm{ml}$ in male rats [37]. In another study, it has revealed that Sesame oil showed beneficial influence on blood profile including blood glucose and glycosylated hemoglobin in rats with diet containing $2 \mathrm{ml}$ of oil [38]. In an experiment, 
it has been showed that Sesame oil with $20 \%$ of feed intake has showed a protective effect in the brain to enhance the immunity of rats against the oxidative stress [39].

Similarly, the results of my work match with various studies to indicate the effects of Clove oil. It was observed that Clove oil has enhanced the feed intake and body weight gain as a natural growth promoter and raised the antibody titer level in broiler chickens at dose concentrations $0.15 \mathrm{ml}, 0.3 \mathrm{ml}$ and $0.45 \mathrm{ml}$ [40]. In another study, Clove oil has improved the fitness status, meat quality, blood components and reduced the intestinal pathogens in quails at different concentrations $(0.75 \mathrm{ml}$ and $1.5 \mathrm{ml} / \mathrm{kg})$ [41]. It has been identified that Clove oil has improved the immune response and activated relaxation signal pathway for more immunity enhancement in rats [42]. It was also observed that dietary supplements with $0.5 \%$, $1 \%$ and $1.5 \%$ of clove oil were useful for growth, disease resistance and strong immunity in Nile tilapia [43].

The combined use of the Sesame oil and Clove oil has also shown better results. The weight gain, blood profile and immunity has extended to high level by oils effects collectively, than separate nutrient did.

\section{Conclusion}

Sesame oil and Clove oil have significantly increased $(\mathrm{p}<0.05)$ the growth performance, blood profile and immunity of male rabbits. The addition of these oils in the diet of rabbits stimulated their digestive activity and resulted as a growth promoter. However, the specific concentration of both oils showed the positive result as; sesame oil promoted the weight, hematological parameters and immunity at $0.3 \mathrm{ml} / \mathrm{kg}$ dose concentration, while clove oil enhanced the weight, blood components and immunity at $0.2 \mathrm{ml} / \mathrm{kg}$ dose concentration. Sesame oil was more active than clove oil in enhancing the parameters during the whole experiment. Furthermore, $\mathrm{SO}+\mathrm{CO}$ at concentrations $0.3+0.3 \mathrm{ml} / \mathrm{kg}$ body weight showed the better results in improving the health of male rabbits.

\section{Authors' contributions}

Conceived and designed the experiments: M Iqbal \& R Iqbal, Performed the experiments: M Iqbal, Analyzed the data: M Hussain, A Razaq, MF Malik \& M Hussain, Contributed reagents/materials/ analysis tools: M Iqbal \& R Iqbal, Wrote the paper: M Iqbal \& R Iqbal. Acknowledgement

Authors are extremely grateful to Almighty Allah and Prophet Muhammad (Heartfelt thanks to Parents and all Respected Teachers for their kind behavior, assistance and guidance. This research did not get any fund from funding agencies in public, marketable and non-profit sectors. The authors acknowledge the support given by Laboratory staff of Department of Zoology, Univeristy of Gujrat.

\section{References}

1. Kalemba D \& Kunicka A (2003). Antibacterial and antifungal properties of essential oils. Curr Med Chem 10: 813829.

2. Bento M, Ouwehand A, Tiihonen, K., Lahtinen S, Nurminen P, Saarinen M \& Fischer J (2013). Essential oils and their use in animal feeds for monogastric animals - Effects on feed quality, gut microbiota, growth performance and food safety: A review. Vet Med 58(9): 449458.

3. Prakash B, Singh P, Kedia A \& Dubey NK (2012). Assessment of some essential oils as food preservatives based on antifungal, antiaflatoxin, antioxidant activities and in vivo efficacy in food system. Int Food Res J 49: 201-208.

4. Saydut A, Duz MZ, Kaya C, Kafadar AB \& Hamamci C (2008). Transesterified sesame (Sesamum indicum L.) seed oil as a biodiesel fuel. Bioresour Technol 99: 6656-6660.

5. Zeb A, Muhammad B \& Ullah F (2017). Characterization of sesame (Sesamum 
indicum L.) seed oil from Pakistan for phenolic composition, quality characteristics and potential beneficial properties. J Food Meas Charact 11(3): 1362-1369.

6. Pathak N, Bhaduri A \& Rai AK (2019). Sesame: Bioactive Compounds and Health Benefits. Bioact Mol in Food 181200.

7. Nayar NM \& Mehra KL (2002). Sesame: Its uses, botany, cytogenetics, and origin. Econ Bot 24: 20-31.

8. Elleuch M, Besbes S, Roiseux O, Blecker C \& Attia H (2007). Quality characteristics of sesame seeds and byproducts. Food Chem 103: 641-650.

9. Prasad MNN, KRS \& Prasad SD (2012). A Review on Nutritional and Nutraceutical Properties of Sesame. J Nutr Food Sci 02(02).

10. Reshma MV, Balachandran C, Arumughan C, Sunderasan A \& Sukumaran D (2010). Extraction, separation and characterisation of sesame oil lignan for nutraceutical applications. Food Chem 120: 1041-1046.

11. Dalal I, Goldberg M \& Katz Y (2012). "Sesame seed food allergy". Curr Allergy Asthma Rep 12(4): 339-45.

12. Morris JB (2002). Food, industrial, nutraceutical, and pharmaceutical uses of sesame genetic resources. In: Janick J, Whipkey A, editors. Trends in new crops and new uses. Alexandria, VA: ASHA Press. 153-156.

13. Chaieb K, Hajlaoui H, Zmantar T, KahlaNakbi AB, Rouabhia M, Mahdouani K \& Bakhrouf A (2007). The chemical composition and biological activity of clove essential oil, Eugenia caryophyllata (Syzigium aromaticum L. Myrtaceae): a short review. Phytother Res 21(6): 501-506.

14. Prashar A, Locke IC \& Evans CS (2006). Cytotoxicity of clove (Syzygium aromaticum) oil and its major components to human skin cells. Cell Proliferation 39: 241-248.

15. Pawar V C, \& Thaker V S (2006). In vitro efficacy of 75 essential oils against Aspergillus niger. Mycoses 49: 316-323.

16. Feres M, Figueiredo LC, Barreto IM, Coelho MN, Araujo MW \& Cortelli SC (2005). In vitro antimicrobial activity of plant extracts and propolis in saliva samples of healthy and periodontallyinvolved subjects. J Int Acad Periodontol 7: 90-96.

17. Manohar V, Ingram C \& Gray J (2001). Antifungal activities of origanum oil against Candida albicans. Mol Cell Biochem 228: 111-117.

18. Ogata M, Hoshi M, Urano S \& Endo T (2000). Antioxidant activity of eugenol and related monomeric and dimeric compounds. Chem Pharm Bull 48: 14671469.

19. Atsumi T, Iwakura I, Fujisawa $S$ \& Ueha $T$ (2001). Reactive oxygen species generation and photo-cytotoxicity of eugenol in solutions of various $\mathrm{pH}$. Biomaterials 22: 1459-1466.

20. Miyazawa M \& Hisama M (2001). Suppression of chemical mutagen induced SOS response by alkylphenols from clove (Syzygium aromaticum) in Salmonella typhymurium TA1535/pSK1002 umu test. J Agric Food Chem 49: 4019- 4025.

21. Roubach R, Gomes LC, Leao Fonseca FA \& LuizVal A (2005). Eugenol as an efficacious anaesthetic for tambaqui, Colossoma macropomum (Cuvier). Aquac Res 36: 1056- 1061.

22. Yadav R \& Yadav SK (2013). Dental disease and its cure- a review, Asian $J$ Pharm and Clin Res 6(2): 1620.

23. Singh J, Baghotia A \& Goel SP (2012). Eugenia caryophyllata Thunberg (Family Myrtaceae): A Review. Int J Res Pharm Biomed sci 3(4): 1469-1475. 
24. Dashti-RHM \& Morshedi A (2009). The effects of Syzygium aromaticum (clove) on learning and memory in mice. Asian J Tradit Med 4(4): 128-133.

25. Pulikottil S \& Nath S (2015). Potential of clove of Syzygium aromaticum in development of a therapeutic agent for periodontal disease: a review. $S$ Afr Dent J 70(3): 108-115.

26. Agrawal M, Agrawal S, Rastogi DR, Singh D P, BR D A \& Gupta D H L (2014). A review on uses of clove in oral and general health. Indian J Res Pharm Biotech 2(4): 1323.

27. Bradbury AG \& Dickens GJE (2016). Appropriate handling of pet rabbits: a literature review. J Small Anim Pract 57(10): 503-509.

28. Ahmad HS, Razooqi AL-Janabi QA \& Shaban AL-Qesee RK (2019). Study of the therapeutic role against diabetes for olive oil, soybean oil and sesame oil on some standards of male reproductive system of local rabbits. Res $J$ Pharm Technol 12: 9-10.

29. Naeem S, Ali L, Rizwani GH, Ikram R, Khan SS, Shareef H, Younus I, Malick TZ \& Aleem U (2020). A comparative neurobehavioral study of sesame oil and fish oil on experimental animals. Pak $J$ Pharm Sci 33(2): 511-521.

30. Gidenne T, Garreau H, Drouilhet L, Aubert C \& Maertens L (2017). Improving feed efficiency in rabbit production, a review on nutritional, technico-economical, genetic and environmental aspects. Anim Feed Sci Technol 225: 109-122.

31. Syrvatka V, Rozgoni I, Slyvchuk Y, Milovanova G, Hevkan I \& Matyukha I (2014). Effects of Silver Nanoparticles in Solution and Liposomal Form on some Blood Parameters in Female Rabbits during Fertilization and Early Embryonic Development. J Microbiol, Biotechnol Food Sci 3(4): 274-278.
32. Parasuraman S, Raveendran R \& Kesavan R (2017). Blood sample collection in small laboratory animals. $J$ Pharmacol Pharmacother 8(3): 153.

33. Reid SA, Speedy DB, Thompson JM, Noakes TD, Mulligan G, Page T \& Milne C (2004). Study of hematological and biochemical parameters in runners completing a standard marathon. Clin J Sport Med 14(6): 344-353.

34. Rehman K, Akash M, Azhar S, Khan S, Abid R, Waseem A \& Sherazi T (2012). A biochemical and histopathologic study showing protection and treatment of gentamicin-induced nephrotoxicity in rabbits using vitamin c. Afr $J$ Tradit Complement Altern Med 9(3).

35. Waltari E, Carabajal E, Sanyal M, Friedland N \& McCutcheon KM (2020). Adaption of a conventional ELISA to a 96-well ELISA-array for measuring the antibody responses to influenza virus proteins, viruses and vaccines. J Immunol Methods 481-482.

36. Abdel-Magid SS, Omer HAA, Abedo AA, Mohamed MI \& Awadalla IM (2009). Partial Substitution of Soybean Meal by Linseed Meal or Sesame Meal in Growing Rabbit's Diet. Egyptian $J$ Nutrition and Feeds 12(3): 503-518.

37. AL-Sallami ASM (2017). Effect of sesame oil on male rats treated with Acrylamide in some physiological and hormonal blood criteria. Int $J$ Curr Pharm Rev Res 8(2): 134-140.

38. Ramesh B, Saravanan R \& Pugalendi K (2005). Influence of sesame oil on blood glucose, lipid peroxidation, and antioxidant status in Streptozotocin diabetic rats. J Med Food 8(3): 377-381.

39. Ahmad S, Yousuf S, Ishrat T, Khan MB, Bhatia K, Fazli IS, Khan JS, Ansari NH \& Islam F (2006). Effect of dietary sesame oil as antioxidant on brain hippocampus of rat in focal cerebral ischemia. Life Sci 79(20): 1921-1928. 42. 
40. Mehr MA, Hassanabadi A, Moghaddam HN \& Kermanshahi H (2014). Supplementation of clove essential oils and probiotic on blood components, lymphoid organs and immune response in broiler chickens. Res Opin Anim Vet Sci 4(4): 218-223.

41. Hussein MM, Abd El-Hack ME, Mahgoub S A, Saadeldin IM \& Swelum AA (2019). Effects of clove (Syzygium aromaticum) oil on quail growth, carcass traits, blood components, meat quality, and intestinal microbiota. Poult Sci 98(1): 319-329.
42. Yilmaz-Oral D, Onder A, Gur S, Carbonell-Barrachina ÁA, KayaSezginer E, Oztekin CV \& Zor M (2020). The beneficial effect of clove essential oil and its major component, eugenol, on erectile function in diabetic rats. Andrologia 52(6).

43. Brum A, Pereira SA, Owatari MS, Chagas EC, Chaves F C, Mouriño JL \& Martins ML (2017). Effect of dietary essential oils of clove basil and ginger on Nile tilapia (Oreochromis niloticus) following challenge with streptococcus agalactiae. Aquacul 468: 235-243. 